\title{
COVID-19 and Kidney Disease: Update on Epidemiology, Clinical Manifestations, Pathophysiology and Management
}

\author{
Masoumeh Asgharpour ${ }^{1}$, Elham Zare ${ }^{2}$, Muhammed Mubarak ${ }^{3}$ and Amirhesam Alirezaei ${ }^{4}$ \\ ${ }^{1}$ Department of Nephrology, Rouhani Hospital, Babol University of Medical Sciences, Babol, Iran \\ ${ }^{2}$ Department of Internal Medicine, North Khorasan University of Medical Sciences, Bojnurd, Iran \\ ${ }^{3}$ Javed I Kazi Department of Histopathology, SIUT, Karachi, Pakistan \\ ${ }^{4}$ Department of Nephrology, Shahid Modarres Hospital, Shahid Beheshti University of Medical Sciences, Tehran, Iran
}

\begin{abstract}
Acute kidney injury (AKI) is relatively common in critically ill coronavirus disease 2019 (COVID-19) patients and it increases mortality and prolongs hospital stay. This article aimed to investigate the history, virology, epidemiology, clinical manifestations, pathophysiology and management of COVID-19 disease, in general, and the pathogenetic mechanisms of severe acute respiratory distress syndrome coronavirus 2 (SARS-CoV2)-induced kidney damage, in particular. Keywords like SARS-CoV2, COVID-19, renal impairment, sepsis, viremia, etc. were used to find relevant publications from PubMed, Scopus, Google Scholar, and clinical trials registry websites. According to different studies, kidney involvement in COVID-19 typically occurs in patients who develop acute respiratory distress syndrome (ARDS) or multiorgan failure. The kidney damage in COVID-19 has been shown to be multifactorial, involving direct viral infection, indirect injury by sepsis, hemodynamic alterations, cytokine storm, disseminated intravascular coagulation and other unknown mechanisms. The presence in kidney of angiotensin-converting enzyme 2 (ACE2), a receptor for the virus, has been proven, but few cases of direct viral presence in kidney tissue have been published. Therefore, further studies are needed to investigate the exact mechanisms underlying kidney impairment. Since the development of AKI is one of the important risk factors for mortality in COVID-19 patients, optimal management of AKI may improve the outcomes.
\end{abstract}

Key Words: Acute kidney injury, Angiotensin-converting enzyme, Sepsis, Cytokine storm, Kidney replacement therapy, Coronavirus disease 2019.

How to cite this article: Asgharpour M, Zare E, Mubarak M, Alirezaei A. COVID-19 and Kidney Disease: Update on Epidemiology, Clinical Manifestations, Pathophysiology and Management. J Coll Physicians Surg Pak 2020; 30(JCPSPCR):CR19-CR25 https://doi.org/10.29271/jcpsp.2020.JCPSPCR.CR19.

Human Coronaviruses (HCoVs) are enveloped viruses belonging to the coronaviridae family which are associated with multiple respiratory diseases. ${ }^{1}$ These have a large positive-sense, single-stranded RNA genome. Upto now, the identified HCoVs are HCoV-OC43, HCoV-HKU1, HCoV-229E, and HCoV-NL63. ${ }^{1}$ In addition, severe acute respiratory distress syndrome (SARS)-associated coronavirus (CoV , SARS-CoV) and Middle-East respiratory syndrome CoV (MERS-CoV), which were responsible for the SARS epidemic in 2002 to 2004 and MERS epidemic in 2012, respectively, have been classified as HCoVs.

Correspondence to: Amirhesam Alirezaei, Department of Nephrology, Shahid Modarres Hospital, Shahid Beheshti University of Medical Sciences, Tehran, Iran

E-mail: amirhesam124@gmail.com

Received: May 19, 2020; Revised: May 21, 2020;

Accepted: June 17, 2020

DOI: https://doi.org/10.29271/jcpsp.2020.JCPSPCR.CR19
In December 2019, the outbreak of a novel coronavirus (CoV), SARS-CoV2, from Wuhan City, Hubei Province of China, resulted in more recent coronavirus disease 2019 (COVID-19) pandemic. SARS-CoV-2, a non-segmented, positive-sense RNA virus, belongs to beta-CoVs. For the first time, this virus was isolated and its genomic characteristics were identified within a short period of time on January $7,2020 .{ }^{1}$ The genome of SARS-CoV2, as a bat-origin CoV, has $79.5 \%$ genetic similarity with SARS-CoV, hence its name. This genome translates several proteins, among which, four proteins are essential, including spike (S) glycoprotein, matrix (M), envelope (E), and nucleocapsid ( $N$ ) proteins. S-proteins bind to angiotensin-converting enzyme 2 (ACE2) receptors to enter the host cells. This receptor is mainly found in the respiratory system. ${ }^{2}$ However, the presence of ACE2 receptors in other organs, including gastrointestinal tract, heart, kidneys and brain, has been documented. $^{2-6}$

In patients with COVID-19 infection, upper and lower respiratory tract involvement, e.g. common cold, bronchiolitis, and pneu- 
monia are the principal manifestations..$^{2-6}$ The primary clinical symptoms of COVID-19 patients are fever, dry cough, fatigue or myalgia. ${ }^{7}$ Within one week of development of signs and symptoms of COVID-19, severe illness may develop in some patients, with progression to dyspnea and hypoxemia, which may rapidly lead to acute respiratory distress syndrome (ARDS). ${ }^{6,7}$ While most patients show favourable prognosis, worst outcomes have been reported in elderly patients and patients with cardiovascular diseases, diabetes mellitus, hypertension, cancer, obesity, and chronic respiratory diseases. ${ }^{7,8}$ Higher mortality rates in severe illness are associated with respiratory, hepatic, renal, cardiac, and gastrointestinal complications, ${ }^{7}$ suggesting multiorgan failure in COVID-19 patients. Thus, SARS-CoV-2 affects lungs as well as other organs such as heart, liver, and kidneys; and patients may die from multiorgan failure. ${ }^{8,9}$ Kidney disease most commonly associated with COVID-19 infection is acute kidney injury (AKI) with histopathological alterations such as acute tubular necrosis (ATN) induced in the vast majority of cases by sepsis, dehydration, cytokine storm syndrome (CSS), rhabdomyolysis and hypoxia. ${ }^{2,6}$ It has been reported that the rate of COVID-19 related mortality increases in the presence of kidney diseases, including AKI, chronic kidney disease (CKD), hemodialysis (HD) and renal replacement therapy (RRT). ${ }^{2,6,7}$ On the other hand, specific treatment or vaccines against SARS-CoV2 are not yet available. ${ }^{2,6}$ Currently, there is no specific antiviral agent to manage the COVID-19 patients with renal diseases; and only non-specific and supportive treatment and RRT are available options. ${ }^{9}$

In this descriptive review, we primarily aim to scrutinise the burgeoning literature on the epidemiology, clinical manifestations, pathophysiology and pathogenic mechanisms underlying kidney involvement in SARS-CoV2 infection as well as to update information on different clinical and therapeutic strategies to manage this condition in patients with COVID-19.

The keywords like SARS-CoV2, COVID-19, kidney, renal impairment, ACE), sepsis, viremia, etc. were used to find relevant publications from PubMed, Scopus, Google Scholar search engines, and clinical trials registry websites.

All research and review articles relevant to this topic directly or indirectly were retrieved and scrutinised. From the information given in these papers, the following aspects were drawn out and presented in this descriptive review for the nephrology and nephropathology community.

\section{Clinical manifestations of COVID-19 disease:}

In comparison with SARS-CoV and MERS-CoV diseases, SARS$\mathrm{CoV}-2$ is more contagious and the transmission from humanto-human occurs through direct contact, droplets, or feces. The incubation period has been estimated to be 1 to 14 days (commonly 3 to 7 days). ${ }^{2-7}$ The virus is found not only in respiratory specimens; but also samples from blood, feces, and urine have been reported to be positive on RT-qPCR. ${ }^{2-7}$ The most common manifestations of COVID-19 are like common cold, e.g. fever, dry cough, dyspnea and fatigue. Less common symptoms include chills, sore throat, headaches, myalgia/arthralgia and pleuritic chest pain. Rarely diarrhea, nausea, vomiting, nasal congestion, palpitations, and chest tightness have been recorded in COVID-19 patients. ${ }^{3-5}$ The presence of underlying diseases such as kidney, liver, and cardiovascular diseases, malignant tumors and old age ( $>50$ years) are risk factors for poor prognosis of the illness. ${ }^{7}$ In $80 \%$ of patients, the illness is mild and prognosis is favourable, with spontaneous recovery, while $14 \%$ experience severe illness and about $6 \%$ of infected patients develop critical condition. ${ }^{7}$ In severe and critical cases, multi-organ involvement including kidneys, heart, liver, as well as the immune and gastrointestinal systems, is observed. ${ }^{2,6,7}$ The ARDS, renal failure, heart failure, and arrhythmias are common causes of death in COVID-19 patients. ${ }^{2,6}$ Thus, the potential multi-organ impairment needs to be considered in clinical approaches to prevent and manage COVID-19 patients. ${ }^{7}$

\section{Renal disease in COVID-19 infection:}

The AKI was shown as a common feature of SARS-CoV as well as other HCoV infections, such as, HCoV-229E, HCoV-NL63, HCoV-OC43, and HCOV-HKU1. In some cases of MERS-CoV, AKI with a potential impact on the severity of illness was reported. Previous studies on SARS-CoV and MERS-CoV have shown that the incidence of AKI was 5-15\% among infected patients, which was associated with a high rate of mortality $(60-90 \%))^{2-6}$ Although preliminary studies have reported lower frequency of AKI (3\%-9\%) in patients with COVID-19, a higher incidence of renal abnormalities was revealed based on more recent reports. ${ }^{6-12}$ In a study by Li et al., on renal function of 59 patients with COVID-19 (28 severe cases and 3 deaths), high levels of serum creatinine $(\mathrm{sCr}, 19 \%)$ and blood urea nitrogen (BUN, $27 \%$ ) were initially manifested and $63 \%$ of patients developed proteinuria during hospitalisation. In all severe cases, radiographic involvement of kidneys was confirmed by computed tomography (CT) scan, indicating reduced density of kidneys due to edema and inflammation of the parenchyma. ${ }^{13}$ High rates of proteinuria (44\%) and hematuria $(26.7 \%)$ were also reported among 710 hospitalised patients with SARS-Cov-2 infection in a study by Cheng et al. ${ }^{4}$ In this study, the prevalence of elevated levels of SCr and BUN was $15.5 \%$ and $14.1 \%$, respectively. ${ }^{4}$ Moreover, higher mortality rates have been reported in COVID-19 patients with comorbid conditions or multi-organ complications such as cardiac disease, ARDS, AKI, electrolyte disturbances, proteinuria, hypoproteinemia, and coagulation disorders compared with patients without any comorbidities or complications. ${ }^{7-11}$

\section{COVID-19 infection in patients with pre-existing kidney diseases:}

Preliminary studies suggest that more serious illness occurs with COVID-19 infection in patients with underlying comorbidities such as hypertension, cardiovascular disease, and diabetes who are at higher risk of intensive care unit (ICU) admission or death. ${ }^{2-5}$ On the other hand, the epidemiological data on SARS-CoV-2 infection among patients with pre-existing kidney disease and patients on HD is limited. In a study from one HD 
centre in Wuhan, COVID-19 impact on HD patients was evaluated and it was revealed that while the severity of illness in HD patients was mild in most cases, HD centers remained high-risk areas and HD patients were a population which was highly susceptible to develop COVID-19 infection compared to healthy population. ${ }^{4}$ The results of a meta-analysis on early and preliminarily available data demonstrated that CKD is associated with higher risk of severe COVID-19 infection. ${ }^{6}$ The mortality rate of pneumonia in patients with CKD has been reported to be 14 to 16 times higher than in the healthy population. ${ }^{11}$

It was shown that both pediatric and adult kidney transplant recipients may be affected by SARS-CoV infection in Hong Kong. Similarly, the MERS-CoV infection was reported to affect this group and led to death due to progressive respiratory disease and AKI. Though SARS-CoV-2 infection has been reported in kidney transplant recipients, the risk, presentation, and outcomes are unknown. ${ }^{14}$ The predominant clinical features of patients were respiratory involvement, and fever but other features such as hypoxia, lymphopenia, and high C-reactive protein (CRP) were also observed. ${ }^{8,11,14}$

\section{Pathogenic mechanisms and pathology of SARS-CoV- 2-induced renal injury:}

The clinical manifestations and genomic structure of SARS-CoV-2 are similar to related viruses of MERS-CoV or SARS-CoV, specifically, the later. Thus, the pathological features of COVID-19 can be predicted to be similar to these viruses. ${ }^{2,12,15-17}$ Moreover, SARS-CoV-2 uses the same receptor to invade the human host cells. SARS-CoV-2 can enter the cells via binding the S-proteins to ACE2 receptors. Subsequently, the transmembrane protease serine 2 (TMPRSS2) activity facilitates the entry and spread of virus by interfacing with the ACE2 receptor. ${ }^{2,6}$ The respiratory disease is the most prominent feature of COVID-19 due to high density of ACE2 receptors on several types of human respiratory system cells, including alveolar epithelial cells (the main target), bronchiolar epithelial cellsand lung vascularcells including endothelium and arterial smooth muscle cells. ${ }^{2,6}$ Similarly, non-respiratory manifestations in patients with COVID-19 may be related to the expression of ACE2 receptors in other organs. In addition to lungs, ACE2 receptors are broadly expressed in organs such as kidney (different cells) and heart (myocardial cells). ${ }^{2}$ The presence of ACE2 receptors has been confirmed in the urinary bladder (urothelium), esophagus (epithelium), and ileum (epithelium). ${ }^{6}$ These findings increase the possibility of COVID-19 involvement of multiple organs with resultant complications, e.g. cardiac diseases (myocardial injury, arrhythmias), gastrointestinal manifestations, AKI, and shock along with ARDS in severe cases. ${ }^{2,6}$

In general, the exact pathogenic mechanisms underlying kidney involvement in COVID-19 disease are unknown. However, a number of mechanisms have been postulated; kidney impairment is related to distribution of ACE2 receptors on different cell types of renal tissue including proximal and distal tubular cells, visceral and parietal epithelial cells of glomerulus, as well as components of interlobular arteries of kidney such as smooth muscle cells and endothelium. ${ }^{6}$ It has also been shown that the expression and activity of this receptor in mouse kidney is higher than heart tissue. The exclusive infection of kidney tissue with SARS-CoV-2 has not been reported. Some histopathological alterations such as ATN without any glomerularinvolvement were reported in the post-mortem autopsy of kidney specimens, confirming the potentials of multi-organfailure responseinduced by systemicinflammatory response, rather than specific organ infection. ${ }^{18}$

Researchers have never detected the SARS-CoV RNA successfully from renal tissue of infected patients. In in-vitro studies on cultured human kidney cells (proximal tubular epithelial cells, glomerular mesangial cells and glomerular epithelial cells) infected by SARS-CoV, productive infection was observed only in proximal tubular epithelial cells but not glomerular cells. A transient infection with poor virus production was observed in mesangial cells; whereas, glomerular epithelial cells were not permissive to SARS-CoV infection. ${ }^{15}$ In another study, the possibility of cultured primary kidney epithelial cells to be infected by SARS-CoV and MERS-CoV was evaluated and the cytopathogenic infection in kidney cells was reported only with MERS$\mathrm{CoV} .{ }^{16}$ More recently, the occurrence of viral RNA and viral products in kidney tissue and urine of symptomatic patients of COVID-19 infection has been documented in some studies. ${ }^{17} \mathrm{Su}$ et al. also found viral particles in podocytes and tubular epithelial cells on ultrastructural study of postmortem specimens from kidneys. ${ }^{18}$ Tubulointerstitial compartment is the main target of injury in this disease with the development of ATI, frank ATN and probably tubulointersititial nephritis. A number of case reports have described glomerulopathic lesions in COVID-19 patients. ${ }^{10} \mathrm{~A}$ high expression of ACE2 in kidney, particularly in podocytes and proximal tubular epithelial cells, is a possible link to the susceptibility of renal parenchymal cells and direct viral invasion of kidney parenchyma following COVID-19 infection. 2,6,10

Various factors such as genetic, environmental, and immune factors are important in determining the severity of HCoVsinfection..$^{2 \cdot 5}$ Following infection, the enhanced levels of cytokines/chemokines, critical mediators of inflammatory response following sepsis, lead to cytokine release syndrome (CRS) and lately hyperinflammatory syndrome. ${ }^{2-6}$ Studies have proven the development of CRS following SARS-CoV-2 infection. ${ }^{6}$ Similar complication was also reported in patients with SARS-CoV and MERS-CoV infections. This phenomenon is largely responsible for frequent complications such as ARDS, respiratory failure, hepatic and renal insufficiency, in severe cases of COVID-19, which increase the risk of death in infected patients. ${ }^{2,6}$ Various types of proinflammatory cytokines, e.g., tumor necrosis factor- $\alpha$ (TNF- $\alpha$ ), interleukin (IL)-2, IL-7, IL-6, monocyte chemoattractant protein 1 (MCP-1), interferon- $\gamma$ inducible protein 10 (IP-10, CXCL10), granulocyte-colony stimulating factor (GCSF), and macrophage inflammatory protein $1-\alpha$ 
(MIP)-1 $\alpha$ have been shown to be related to the severity of COVID-19 disease. ${ }^{6,19,20}$ Among these mediators, themostimportant cytokine is pro-inflammatory IL-6. Patients with COVID-19 have high concentrations of cytokines including IL-1 $\beta$, IFN- $\gamma$, IP-10, and MCP, and patients requiring ICU admission have higher levels of IP-10, GCSF, MCP-1, MIP-1 $\alpha$, and TNF- $\alpha{ }^{2,6,19,20}$

\section{Therapeutic and medical approaches:}

Yet, no specific treatment is available for COVID-19 infection. Hence, treatment strategies are aimed at general and supportive measures, as well as the use of empirical therapeutic agents. ${ }^{9,21-23}$ All suspected and confirmed cases should be treated in designated hospitals equipped with effective isolation and protective facilities. Suspected cases should be isolated and treated in separate rooms, and confirmed cases can be treated in the same ward. Importantly, critical cases need to be admitted to the ICU as soon as possible. Here, the therapeutic approaches are briefly reviewed as they relate to renal complications in COVID-19 disease.

\section{Extracorporeal therapy:}

Extracorporeal therapies include various approaches to remove cytokines from the blood of individuals with sepsis and septic AKI and could potentially be beneficial in critically ill patients with COVID-19. ${ }^{9}$ For this purpose, some strategies can be used: direct haemoperfusion (HP), plasma adsorptionon a resinfollowingseparation of plasma, and different methods of continuous renal replacement therapy (CRRT). CRRT was reported to have favourable outcome in patients with SARS-CoV and MERS-CoV infection as well as septic and multiorgan failure conditions. Therefore, CRRT may play a role in patients with COVID-19-related sepsis syndrome. In a retrospective cohort study, the beneficial effect of CRRT on all-cause mortality of patients with COVID-19 undergoing invasive mechanical ventilation was proven. ${ }^{9}$

Nowadays, adsorption with different forms of blood purification, plasma perfusion, or coupled plasma filtration-adsorption has been demonstrated to be popular in treatment of sepsis. In a study on multiple organ dysfunction syndrome patients with serious injury or sepsis with/without acute renal failure, it was demonstrated that blood purification with resin adsorption along with continuous veno-venous hemofiltration could attenuate the levels of proinflammatory cytokines such as IL-6, IL-1 $\beta$, and TNF- $\alpha$. Also, an improvement of clinical symptoms and signs as well as cellular immunity was confirmed in these patients. Recently, the beneficial effects of blood purification in the treatment of severe COVID-19 patients have been discussed in detail. ${ }^{9}$

\section{Antiviral therapy:}

Type 1 interferon (IFN- 1 ) with $\alpha, \beta, \varepsilon$, $\omega$ and $k$ subtypes is considered as one of the most important factors in innate immune system which affects the viral replication during the early phases of infection. Among various subtypes, IFN-1 $\beta$ subtype has been revealed to be most effective for treatment of COVID-19 patients. ${ }^{21}$ Transcription via the IFN activated Janus kinase/signal transducers and activators of transcription (JAK/STAT) signalling cascade results in the overexpression of many IFN-regulated genes that rapidly kill viruses in infected cells.
Although, the efficacy of IFN is unclear due to conflicting findings in SARS-CoV and MERS-CoV infections, novel coronavirus was proven to be much more susceptible to INF type I pretreatment. Additionally, due to the risk of development of nephropathy by treatment with IFN in several cases, the clinicians should be careful in administration of this drug for COVID-19 patients with renal failure. ${ }^{21}$

Direct antiviral agents including five FDA-approved medicines (ribavirin, penciclovir, nafamostat, nitazoxanide, and chloroquine) and two experimental drugs (remdesivir and favipiravir) have been recommended. ${ }^{22}$ In an in-vitrostudy, remdesivir, lopinavir, emetine, and homoharringtonine were confirmed to inhibit the replication of SARS-CoV-2. In a multicenter trial, the potential efficacy of chloroquine phosphate, antimalarial and anti-inflammatory drug, against SARS-CoV-2 infection was demonstrated. Nevertheless, some conflicting data on efficacy of chloroquine phosphate in COVID-19 patients have also emerged necessitating caution in its use. ${ }^{22}$

Additionally, the safety and tolerability of ribavirin in the treatment of viral infection (Hepatitis C) in patients with kidney disease has been evaluated and $200 \mathrm{mg} /$ day for severe and the 200 or 400 mg/day doses for moderate renal impairment were suggested. The adverse effects of nitazoxanide $(18 \mathrm{mg} / \mathrm{kg})$ and chloroquine $(50 \mathrm{mg} / \mathrm{kg})$ on kidney were proven in an animal model. ${ }^{22}$ There is no report to indicate the safety of other antiviral agents such as penciclovir and nafamostat in COVID-19 patients with renal impairment. As no specific drug has been developed for the treatment of COVID-19-induced nephropathy, the proposed antiviral agents which reduce the viremia and manage sepsis, may also be beneficial in critically ill patients.

\section{Immunosuppressions:}

Corticosteroids: Treatment of COVID-19 patients with corticosteroids is a double-edged sword and due to the potential prolongation of virus shedding and increased duration of viremia, the administration of corticosteroids remains controversial and is not recommended by WHO. Clinical evidence from SARS, MERS and other severe respiratory virus infections does not support the use of corticosteroids to treat COVID-19 patients with lung injury or shock. ${ }^{23}$ The results of a retrospective observational study in MERS patients showed that high-dose steroids probably increased demand for vasopressors, mechanical ventilation, and RRT. ${ }^{23}$

In a retrospective study of SARS-CoV infected patients, corticosteroid administration in non-critical patients was accompanied by a survival rate of $100 \%$, and in critical cases, reduced mortality rate and shortened hospital stay was reported, suggesting administration of proper doses of corticosteroids as beneficial in carefully selected critical cases. In another relevant research, low to moderate doses of corticosteroids reduced mortality in patients with influenza A(H1N1) pdm09-associated respiratory diseases and oxygen index lower than 300 $\mathrm{mm} \mathrm{Hg}$. In a recently published article, a proper guideline with a 
short course of low to moderate doses of corticosteroids for critically ill COVID-19 patients was suggested. ${ }^{23}$

\section{Nonsteroidal anti-inflammatory drugs (NSAIDs):}

NSAIDs were considered as a treatment option in COVID-19 patients. In an in-vitro study, indomethacin, most prescribed medicine for treatment of arthritis and gout, was shown to exhibit antiviral activity against SARS-CoV (dose of $1 \mathrm{mg} / \mathrm{kg}$ ) by inhibition of virus replication. Furthermore, the antiviral effects of naproxen have been proved against influenza A and B in animal studies. This drug exhibits its efficacy by interfering with the process of RNA replication. In an under-processed clinical trial, the efficacy of naproxen supplementation in the treatment regimen of critically ill patients with COVID-19 is being investigated in Hôpitaux de Paris (NCT04325633). Since SARS-CoV2 is a single-stranded RNA virus, it has been reported that naproxen may have the potential to manage the outbreak of novel CoV infection.

The increased levels of ACE2 can facilitate infection with these viruses. The expression of ACE2 has been shown to be increased through the administration of ibuprofen as an NSAID in diabetic patients and in those treated with blockers of angiotensin II type-I receptors. ${ }^{23}$

\section{Janus kinase (JAK) inhibitors:}

Baricitinib, ruxolitinib, and fedratinib, as selective inhibitors of JAK pathway, were approved for treatment of rheumatoid arthritis and myelofibrosis. The potential use of baricitinib for treatment of COVID-19 is under doubt. This agent targets the numb-associated kinase (NAK) family, with a high affinity for AAK1 and inhibits the clathrin-mediated endocytosis regulated by NAK and decreases viral infection in some in-vitro studies. Because of high efficacy of the drug against the enhanced levels of cytokines in conditions such as SARS-CoV2 infection, baricitinib in combination with antiviral agents, was proposed to be used in treatment of COVID-19 patients. ${ }^{22}$

\section{Monoclonal antibodies:}

A specific monoclonal antibody against SARS-CoV-2 infection has not yet been introduced. TNF receptors are related to a superfamily of cytokines and inhibition of these receptors may modulate the inflammatory responses. TNF- $\alpha$, as a member of cytokines, demonstrates proinflammatory activity. Therefore, TNF- $\alpha$ blocker can be considered as a potential therapeutic approach in the treatment of autoimmune and immune-mediated disorders. ${ }^{22}$ In previous studies, this drug has also been used in the management of SARS-CoV patients with severe immunebased pulmonary injury. However, anti-TNF- $\alpha$ therapy did not ameliorate disease in an animal model of acute virus-endotoxin respiratory disease in pigs. There is no evidence to show the safety and efficacy of anti-TNF- $\alpha$ monoclonal antibody against COVID-19. IL-6, a prominent cytokine in the inflammatory responses, can be targeted because of the dysregulation and overexpression during the inflammatory disease. Tocilizumab (anti-IL-6 monoclonal antibody) has been recently proposed to be used in the treatment of patients with severe COVID-19.
Overall, there is no solid evidence to show the effectiveness of anti- IL-6 on the modulation of COVID-19. ${ }^{22}$

\section{Stem cell therapy:}

Stem cell therapy as a type of cell therapy has been used widely in the treatment of different diseases in basic and clinical studies. ${ }^{12,24}$ Among various types of stem cells, mesenchymal stem cells (MSCs) with beneficial characteristics such as minimal invasive features, high rate of proliferation, and powerful immunomodulatory activity, and safety and lack of ethical issues, are attracting researchers' attention in the treatment of different diseases. The clinical application of MSCs have been well documented in inflammatory diseases, e.g. systemic lupus erythematosus and graft versus-host disease (GVHD). MSCs can induce their therapeutic effects through two strategies; suppression of inflammatory responses and differentiation into the cells of host tissue. ${ }^{24}$ The immunomodulatory features of MSCs are related to their direct interactions with immune cells and secretion of many types of cytokines. Toll-like receptors (TLRs), which are activated by pathogen-related molecules, e.g., endotoxin or double-stranded RNA of viruses, stimulate the MSCs to trigger furtherimmunomodulatory effects.

Currently, MSCs have been used in the treatment of COVID-19 patients. For this purpose, Leng et al. evaluated the effects of transplanted MSCs in COVID-19 patients with pneumonia and reported that MSCs, as a source of stem cells, with proven features of ACE2- and TMPRSS2-, were safe and successful in the treatment of COVID-19 pneumonia, particularly in severe cases. ${ }^{12}$ Umbilical cord MSCs have also been suggested to be used as a therapeutic strategy in managing critically ill COVID-19 patients.

\section{Convalescent plasma therapy:}

Convalescent plasma (CP) or immunoglobulins have been approved by FDA to be used in treatment of COVID-19 patients. In several studies, CP has been shown to reduce the duration of hospital stay and mortality rate in SARS patients treated with CP. In 2015, a protocol of CP was established for treatment of MERS$\mathrm{CoV}$ infectious disease. Recently, CP also has been proposed to be used in the treatment of COVID-19 patients. In this preliminary uncontrolled case series, the CP therapy containing neutralizing antibody was successful in treatment of 5 critically ill COVID-19 patients through neutralizing viremia and possibly regulating the cytokine storm. In a similar study, 10 severe cases were investigated and one dose of $\mathrm{CP}(200 \mathrm{~mL})$ was transfused. According to the results, CP therapy was safe and effectively improved the clinical outcomes of severe COVID-19 cases with no severe adverse events. Moreover, the findings of CP therapy in 6 laboratory confirmed COVID-19 patients in Wuhan, indicated that this approach was specifically effective in eliminating the SARS-CoV-2, considering it as the most promising therapy for COVID-19 pandemic. It has been suggested that CP could be considered in individuals with early symptoms of COVID-19 to prevent disease progression. ${ }^{25} \mathrm{At}$ present, two trials have been recorded to evaluate the efficacy and safety of CP in patients with COVID-19 as an open-label, nonrandomised clinical trials in 
Wuhan Union Hospital, China (NCT04264858) and a prospective randomized controlled trial in China (ChiCTR2000029757).

\section{CONCLUSION}

There is a definite risk of renal injury in some patients with COVID-19 disease, but to date only preliminary information is available. AKI and early proteinuria and hematuria are observed in a subset of patients with COVID-19, but the available data is conflicting and varied. Among these, the development of AKI is significant and leads to enhanced mortality. Although the exact pathogenic mechanism are yet unclear, but the kidney involvement is most likely, multifactorial in origin. Tubulointerstitial compartment is the main target of injury in this disease with the development of ATI, frank ATN and probably tubulointersititial nephritis. More recently, a number of case reports have described glomerulopathic lesions in COVID-19 patients. On the other hand, patients with CKD, renal transplant patients and HD patients are at increased risk of contracting and developing severe form of COVID-19 infection. There is a need for further research on kidney involvement in COVID-19 disease so that effective strategies for prevention and early management of AKI can be instituted. It should be kept in mind that prevention of AKIthrough precise control offluid and electrolyte balance, avoidance of nephrotoxic drugs and daily monitoring of kidney function and urinary output, is of utmost importance in improving the outcome of COVID-19 patients.

\section{CONFLICT OF INTEREST:}

Authors declared no conflict of interest.

\section{AUTHORS' CONTRIBUTION:}

MA: Data acquisition and analysis, interpretation, drafting, final approval.

EZ: Conception and design, Interpretation, drafting, final approval.

MM: Critical revision, final drafting, final approval.

$A A$ : Conception and design, interpretation, critical revision, final drafting, final approval.

\section{REFERENCES}

1. Lu R, Zhao X, Li J, Niu P, Yang B, Wu H, et al. Genomic characterisation and epidemiology of 2019 novel coronavirus: Implications for virus origins and receptor binding. Lancet 2020; 395(10224):565-74.

2. Tolouian R, Vahed SZ, Ghiyasvand S, Tolouian A, Ardalan M. COVID-19 interactions with angiotensin-converting enzyme 2 (ACE2) and the kinin system; looking at a potential treatment. J Renal Inj Prev 2020; 9(2): e19.

3. Huang C, Wang Y, Li X, Ren L, Zhao J, Hu Y, et al. Clinical features of patients infected with 2019 novel coronavirus in Wuhan, China. Lancet 2020; 395(10223):497-506.

4. Cheng Y, Luo R, Wang K, Zhang M, Wang Z, Dong L, et al. Kidney disease is associated with in-hospital death of patients with COVID-19. Kidney Int 2020; 97(5):829-38.

5. Wang D, Hu B, Hu C, Zhu F, Liu X, Zhang J, et al. Clinical characteristics of 138 hospitalised patients with 2019 novel coronavirus-infected pneumonia in Wuhan, China. JAMA
2020; 323(11):1061-9.

6. Mubarak M, Nasri N. COVID-19 nephropathy: An emerging condition caused by novel coronavirus infection. J Nephropathol 2020; 9(3):e21.

7. Zhou F, Yu T, Du R, Fan G, Liu Y, Liu Z, et al. Clinical course and risk factors for mortality of adult inpatients with COVID-19 in Wuhan, China: A retrospective cohort study. Lancet 2020; 395(10229):1054-62.

8. Alberici F, Delbarba E, Manenti C, Econimo L, Valerio F, Pola $A$, et al. Management of patients on dialysis and with kidney transplant during SARS-CoV-2 (COVID-19) pandemic in Brescia, Italy. Kidney Int Rep 2020; 5(5):580-5.

9. Ronco C, Reis T. Kidney involvement in COVID-19 and rationale for extracorporeal therapies. Nat Rev Nephrol 2020; 16(6):308-10.

10. Mubarak M, Tolouian R, Pezeshgi A. Collapsing glomerulopathy following COVID-19 infection; possible relationship with APOL1 kidney risk alleles in AfricanAmericans. Immunopathol Persa 2020; 6(2):e18.

11. Ma Y, Diao B, Lv X, Zhu J, Liang W, Liu L, et al. 2019 novel coronavirus disease in hemodialysis (HD) patients: Report from one HD center in Wuhan, China. MedRxiv 2020.

12. Leng Z, Zhu R, Hou W, Feng Y, Yang Y, Han Q, et al. Transplantation of ACE2-mesenchymal stem cells improves the outcome of patients with COVID-19 pneumonia. Aging Dis 2020; 11(2):216-28.

13. Li Z, Wu M, Yao J, Guo J, Liao X, Song S, et al. Caution on kidney dysfunctions of COVID-19 patients. MedRxiv 2020;

14. Banerjee D, Popoola J, Shah S, Ster IC, Quan V, Phanish M. COVID-19 infection in kidney transplant recipients. Kidney Int 2020; 97(6):1076-82.

15. Tang JW, To KF, Lo AW, Sung JJ, Ng HK, Chan PK. Quantitative temporal-spatial distribution of severe acute respiratory syndrome-associated coronavirus (SARS-CoV) in post-mortem tissues. J Med Virol 2007; 79(9):1245-53.

16. Pacciarini F, Ghezzi S, Canducci F, Sims A, Sampaolo M, Ferioli $E$, et al. Persistent replication of severe acute respiratory syndrome coronavirus in human tubular kidney cells selects for adaptive mutations in the membrane protein. J Virol 2008; 82(11):5137-44.

17. Peng L, Liu J, Xu W, Luo Q, Deng K, Lin B, et al. 2019 novel coronavirus can be detected in urine, blood, anal swabs and oropharyngeal swabs samples. J Med Virol 2020; 10.1002/jmv.25936.

18. Su H, Yang M, Wan C, Yi LX, Tang F, Zhu HY, et al. Renal histopathological analysis of 26 postmortem findings of patients with COVID-19 in China. Kidney Int 2020; 98(1):219-27.

19. Argani H, Alirezaei A, Ghorbanihaghjo A, Azizi T, Asgharpour M, Bakhtiyari M. Comparing the serum levels of adipocytokines in the renal transplant recipients and healthy individuals: A case-control study. Iran Red Crescent Med J 2018; 20(3):e62674.

20. Naji HE, Ghorbanihaghjo A, Argani H, Raeisi S, Safa J, Alirezaei $A$, et al. Serum STWEAK and FGF-23 levels in hemodialysis and renal transplant patients. Int J Organ Transplant Med 2017; 8(2):110-16.

21. Sallard E, Lescure FX, Yazdanpanah Y, Mentre F, Peiffer- 
Smadja N, Committee CDFS. Type 1 interferons as a potential treatment against COVID-19. Antiviral Res 2020; 178:104791.

22. Valizadeh R, Dadashzadeh N, Zakeri R, James Kelllner S, Rahimi MM. Drug therapy in hospitalized patients with very severe symptoms following COVID-19. J Nephropharmacol 2020; $9(2): e 21$.

23. Russell B, Moss C, Rigg A, Van Hemelrijck M. COVID-19 and treatment with NSAIDs and corticosteroids: Should we be limiting their use in the clinical setting? Ecancermedicalscience 2020; 14:1023.

24. Golchin A, Seyedjafari E, Ardeshirylajimi A. Mesenchymal stem cell therapy for COVID-19: Present or future. Stem Cell Rev Rep 2020; 16(3):427-33.

25. Duan K, Liu B, Li C, Zhang H, Yu T, Qu J, et al. Effectiveness of convalescent plasma therapy in severe COVID-19 patients. Proc Natl Acad Sci USA 2020; 117(17):9490-6. 